•综述・

\title{
葡萄座腔菌科真菌的系统学和多样性探讨
}

\author{
李文英 ${ }^{1,2,3^{*}}$ 李 夏 ${ }^{1}$ 解开治 ${ }^{1}$ 邓旺秋 ${ }^{2}$ 庄文颖 ${ }^{3}$ \\ 1 (广东省农业科学院农业资源与环境研究所农业部南方植物营养与肥料重点实验室/广东省养分资源循环利用 \\ 与耕地保育重点实验室, 广州 510640) \\ 2 (广东省微生物研究所省部共建华南应用微生物国家重点实验室, 广州 510070) \\ 3 (中国科学院微生物研究所真菌学国家重点实验室, 北京 100101)
}

\begin{abstract}
摘要: 葡萄座腔菌科的真菌是子囊菌中重要的代表性类群, 是农林生态系统中重要的组成部分。该类真菌物种资 源丰富、全型特征多样, 包括许多具有重要经济价值和生态功能的类群, 隶属子囊菌门(Ascomycota)果囊菌亚门 (Pezizomycotina)座囊菌纲(Dothideomycetes)葡萄座腔菌目(Botryosphaeriales)。本文在概述葡萄座腔科真菌生态功 能的基础上, 回顾了一百年来该类真菌目、科、属和种各级分类单元的建立及其系统分类研究历史, 重点评述以 von Arx \& Müller和Barr提出的两大分类系统, 总结了基于全型形态特征和多基因序列分析的现代分子系统学研 究现状。虽然该类群的分类系统日趋完善, 但中国的相关研究存在资源挖掘不够深入、研究的系统性不足等问题。 主要针对资源挖掘必要性、分类依据科学性、物种特征全息化等几个方面存在的问题进行了分析和讨论, 并对未 来研究方向进行展望，以期为该类真菌的深入研究与开发利用提供科学依据。
\end{abstract}

关键词：葡萄座腔菌目；葡萄座腔菌科；系统分类学；生物多样性；系统发育

\section{Systematics and species diversity of botryosphaeriaceous fungi}

Wenying $\mathrm{Li}^{1,2,3^{*}}$, Xia Li ${ }^{1}$, Kaizhi Xie ${ }^{1}$, Wangqiu Deng ${ }^{2}$, Wenying Zhuang ${ }^{3}$

1 Key Laboratory of Plant Nutrition and Fertilizer in South Region, Ministry of Agriculture/Guangdong Key Laboratory of Nutrient Cycling and Farmland Conservation, Institute of Agricultural Resources and Environment, Guangdong Academy of Agricultural Sciences, Guangzhou 510640

2 State Key Laboratory of Applied Microbiology Southern China, Guangdong Institute of Microbiology, Guangzhou 510070

3 State Key Laboratory of Mycology, Institute of Microbiology, Chinese Academy of Sciences, Beijing 100101

\begin{abstract}
Botryosphaeriaceous fungi are an important ascomycete group (Botryosphaeriales, Dothideomycetes, Pezizomycotina, Ascomycota). They play essential roles in economic and ecological environments, especially in agroforestry ecosystems. In this overview, we introduce a wide variety of ecological functions of botryosphaeriaceous fungi. The taxonomic studies of the family Botryosphaeriaceae in its early stages have been reviewed in details since 1823, especially the two major classification systems proposed by von Arx \& Müller and Barr. The research status, progress based on holomorphological characteristics and multilocus DNA sequence data are summarized towards updating our understanding of species diversity and classification systems of the group. In addition, the research history, current status and work to be done of the group in China are briefly stated. Problems regarding biodiversity and phylogeny are discussed. Research perspectives are proposed based on taxonomic features, species concepts and exploration of bioresources. This review is expected to provide useful information about effective utilization of fungal resources and control of the harmful effects of botryosphaeriaceous fungi.
\end{abstract}

Key words: Botryosphaeriales; Botryosphaeriaceae; systematic; biodiversity; phylogeny 
囊果和分生孢子果为葡萄状子座型, 子囊双囊壁, 子囊孢子和分生孢子较大, 椭圆形至纺绖形, 许多 是具有重要经济价值和生态功能的类群, 隶属子囊 菌门(Ascomycota)果囊菌亚门(Pezizomycotina)座囊 菌纲 (Dothideomycetes) 葡萄座腔菌目 (Botryosphaeriales) (Kirk et al, 2008; Phillips et al, 2013)。该类真 菌为世界性分布, 寄生或腐生于单子叶植物、双子 叶植物或裸子植物等寄主上, 主要生于木本植物的 树干和枝条, 也发生于草本植物的叶片或茎秆上, 甚至地衣体上。有些为植物内生菌或腐生菌, 在自 然界的生态平衡、物质能量循环中发挥一定作用, 有些则为植物病原菌, 引起许多重要植物病害, 具 有重要的经济影响(Barr, 1987; Denman et al, 2000; Slippers \& Wingfield, 2007)。

本文在分析葡萄座腔菌科真菌生态功能和回 顾分类研究历史的基础上, 总结国内外研究进展, 分析中国研究现状, 并对未来的研究进行展望, 以期为该类真菌的深入研究与开发利用提供科学 参考。

\section{1 生态功能及经济重要性}

葡萄座腔菌科真菌及其相关类群的属和种丰 富、形态各异、生态多样, 分布于除极地以外的所 有地理和气候区域, 营腐生、寄生或内生生活, 在 长势衰弱、濒临死亡或刚刚枯死的木本植物组织上 较为常见, 大多数种类可以在人工培养基上生长 (Denman et al, 2000; Huang et al, 2008)。它们占据着 不同的生态位, 在农林生态系统中行使着各自不同 的生态学功能。

该科真菌有些成员是植物腐生菌, 主要腐生或 附生于各种乔木和灌木植物性基质上, 是农林生态 系统中的重要组成。如色二狍属(Diplodia)真菌的一 些种可有效降解木质素和纤维素, 分解枯枝落叶, 对保护生物多样性和维持生态平衡具有重要的意 义(Sivanesan, 1984; Barr, 1987)。

还有些成员是熟知的植物内生菌, 可产生生物 活性物质, 在生物防治和医药方面具有一定的应用 前景。如毛色二狍属(Lasiodiplodia)的一些种, 具抗 菌活性, 可促进名贵中药沉香的结香过程(韩晓敏 等, 2014; Wei et al, 2014), 对羊蹄甲白粉病菌也具 有拮抗作用(Kumar \& Singh, 2009)。有些种对污染 物具有降解或钝化效应, 在污染环境生物修复方面
具有应用前景(张志远等, 2012; Deng et al, 2014)。

葡萄座腔菌科真菌的更多成员为弱寄生菌, 条 件适宜时易引起植物病害, 对农林生产具有重要的 影响(吴小芹等, 2001; Slippers \& Wingfield, 2007; Cunnington et al, 2009)。特别是葡萄座腔菌属 (Botryosphaeria)、新壳梭狍属(Neofusicoccum)、色 二狍属、小穴壳狍属(Dothiorella)、毛色二狍属等的 优势类群会引起流胶、茎枯、梢枯、枝枯、溃疡、 蒂腐、根腐、炭腐等多种植物病害(Slippers et al, 2007; Chen et al, 2011; Machado et al, 2014; Xu et al, $2015 b$ ), 寄生或腐生于多种作物果实, 引起果腐、环 腐、蕉腐、轮纹等果实病害或采后货架期病害 $(\mathrm{Xu}$ et al, 2015a; Zhou et al, 2015; Huang et al, 2016)。

葡萄座腔菌科及其相关类群的许多成员与人 类生活及农林产业紧密相联, 引起了人们对它们的 密切关注。由于该类真菌历经葡萄座腔菌属(1863)、 葡萄座腔菌科(1918)、葡萄座腔菌目(2006)的分类地 位变化, 广义的葡萄座腔菌类真菌囊括了以葡萄座 腔菌科为主的葡萄座腔菌目中的相关类群, 许多属 和种的归属目前还处于不断修订中。在百余年的研 究历程中(1863-2016), 该类真菌分类地位和包含成 员虽几经变化, 但主要属于葡萄座腔菌科, 本文仅 以狭义的葡萄座腔菌科真菌的系统学和多样性为 主线探讨其研究进展及未来展望。

\section{2 经典分类研究}

\section{1 葡萄座腔菌科的建立及其概述}

葡萄座腔菌科由Theissen和Sydow (1918) 建立, 当时隶属于子囊菌门盘菌纲(Discomycetes)座囊菌 亚纲(Dothideineae)假球壳菌目 (Pseudosphaeriales), 囊括葡萄座腔菌属、暗葡腔菌属(Phaeobotryon)和双 葡腔菌属(Dibotryon)等 3 个属。该科包含形态各异的 一类真菌, 分类和命名等方面的变化多样, 曾有 3,000多个名称与Botryosphaeriaceae相关(http://www. indexfungorum.org/Names/names.asp)。根据最新分 类观点, 目前葡萄座腔菌科被处理为子囊菌门座囊 菌纲下未定亚纲葡萄座腔菌目的模式科, 普遍认可 包括约 20 属约 180 种, 其中葡萄座腔菌属为模式属 (Phillips et al, 2013; Slippers et al, 2013; Dissanayake et al, 2016; 图1)。

\section{2 历史与进展}

葡萄座腔菌科真菌及其相关类群最早的研究 
可以追溯到Fries在 1823 年以球壳狍 (Sphaeria dothidea)为名对葡萄座腔菌 (Botryosphaeria dothidea) 的报道。Cesati和Notaris在1863年建立的葡萄座腔 菌属, 是指一类具球状、双囊壁子囊、能引起木本 植物坏死的子囊菌, 但当时没有指定模式种。1 877 年, Saccardo修订一些前述的种类, 较为详细地描述

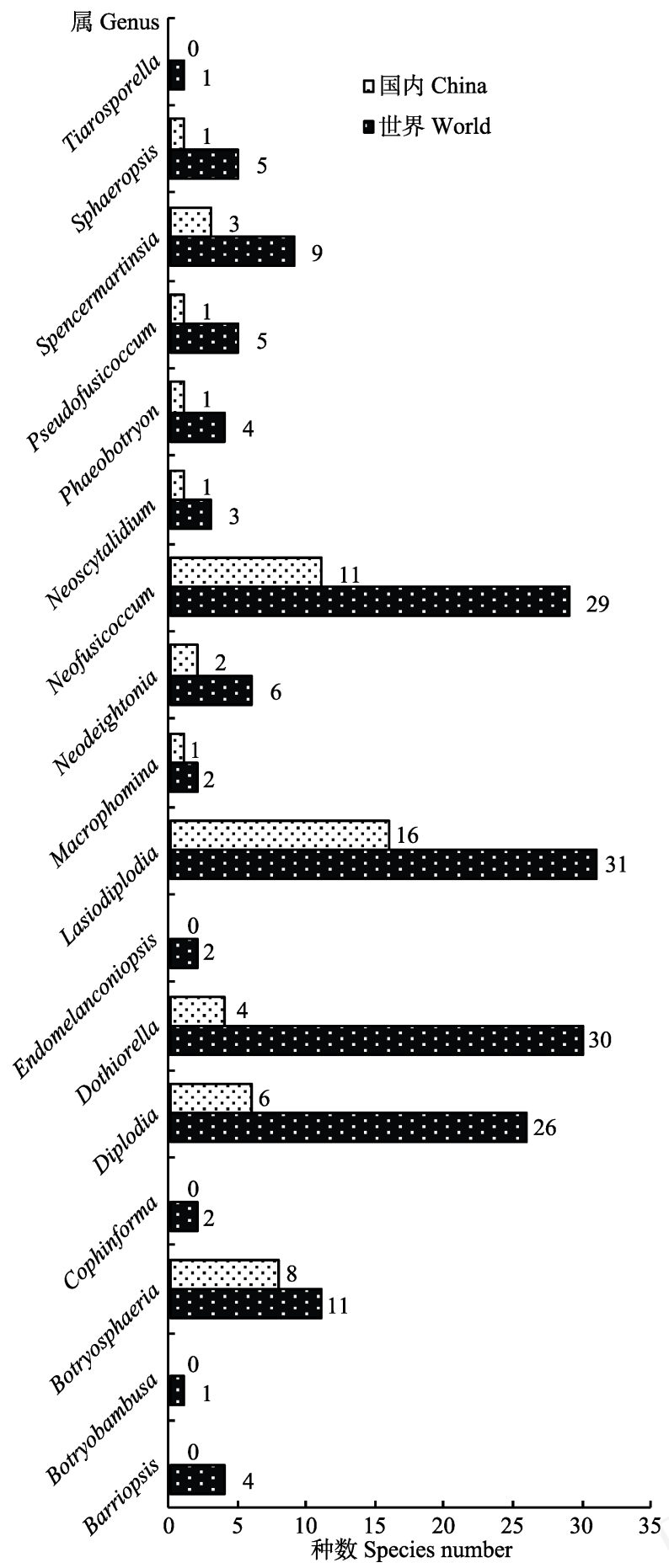

图1 葡萄座腔菌科属种多样性

Fig. 1 Diversity of genera and species in Botryosphaeriaceae
了该属的形态特征。1909年, Höhnel曾建议将B. berengeriana作为该属的后选模式; 而1915年，Theissen和Sydow却建议将B. quercuum作为后选模式。 由于Cesati和De Notaris建立葡萄座腔菌属时没有包 括上述 2 个种，Barr (1972)否定了他们的建议，提出 将葡萄座腔菌作为该属的后选模式种，该提议被后 人普遍采纳。Slippers等(2004)根据新指定的模式和 附加模式明确了葡萄座腔菌的概念, 并给予该属科 学的描述。

Theissen在1916年曾将假球壳菌科归入多腔菌 目(Myriangiales)；1917年，Theissen和Sydow认为假 球壳菌科应该与座囊菌科(Dothideaceae)合并; 1918 年建立座囊菌亚纲，囊括假球壳目葡萄座腔菌科葡 萄座腔菌属。Theissen和Sydow (1918)建立葡萄座腔 菌科时, 曾将该科处理为假球壳菌科(Pseudosphaeriaceae)的一个亚科; Petrak (1923)否定他们的分类处 理，把葡萄座腔菌属归入球壳菌目(Sphaeriales)中 格狍腔菌科(Pleosporaceae)的假球壳菌亚科(Pseudosphaerieae)。

Miller (1928，1938)指出形成子囊果及子囊果 包被的细胞组织存在根本的差异，这些不同的组织 类型与子囊果中心体特征相关联。球壳菌目具典型 子囊壳(perithecial ascomata)和侧丝，而座囊菌目子 囊果为子囊座(ascostromatic ascomata), 无侧丝。由 于葡萄座腔菌属成员缺乏真正的子囊壳包被，所以 从假球壳菌科转入座囊菌目。Nannfeldt (1932)将真 子囊菌纲真菌分为 3 类: 子囊果为闭囊壳类型 (cleistothecial forms), 子囊在子囊果内不规则散生, 归入闭囊壳菌类(plectascales); 子囊座类型 (ascostromatic forms), 子囊在预先形成的子座空腔内形 成, 被归入囊腔菌类(Ascoloculares); 典型的球壳目, 即子囊在子实层内生长发育的类群被归入子囊膜 目(Ascohymeniales)。尽管这些术语当时没有被广泛 接受, 但它们与后来Luttrell(1955)提出的双囊壁 (bitunicate)类群和单囊壁(unitunicate)类群的术语内 涵基本一致。

Luttrell (1951，1955)强调子囊果个体发育特性 在分类上的重要性，提出核菌类(pyrenomycetous fungi)存在 2 种主要形态类型：单囊壁类子囊菌和双 囊壁类子囊菌(腔子囊菌)。Luttrell (1951)还明确指 出 8 种中心体发育类型，强调子囊间不育丝状体组 织的在分类学上的重要性。根据假球壳菌科成员的 
中心体类型将其移入座囊菌目, 至此假球壳菌目不 复存在。Luttrell (1955)用格孢腔菌目(Pleosporales) 取代了假球壳菌目, 其中重要类群葡萄腔菌属成员 具备格狍腔菌型(Pleospora)中心体发育类型, 因此 归入格狍腔菌目。尽管Parguey-Leduc (1966)为葡萄 座腔菌具备典型的格狍腔菌型中心体特征提供了 确業证据, 但Barr (1972)并没有仔细研究子囊间组 织清晰可见的该物种标本, 在她的早期著作中, 仍 将葡萄座腔菌属归入座囊菌目。Barr (1979)之后承 认葡萄座腔菌科具备典型的格孢腔菌型中心体特 征, 从而建立了比较成熟的分类系统, 确定这类真 菌应归入格狍腔菌目, 该目中葡萄座腔菌科包括模 式属葡萄座腔菌属等9个属(Barr, 1983, 1987)。上述 观点得到一些学者的支持, 如Eriksson (1981)强调 葡萄座腔菌科主要成员中心体具备假囊壳和假侧 丝等。

von Arx和Müller (1954，1975)对葡萄座腔菌科 与座囊菌科等相近科之间的区别特征进行了讨论, 指出Luttrell (1955, 1973) 和Barr (1972) 承认的葡萄 座腔菌科是由许多没有关联的属组成, 对部分类群 的归属处理不当, 采用的分类学特征存在一些重叠 与交叉, 如球座菌属(Guignardia)和葡萄座腔菌属 是近似属, 不应该将它们归入座囊菌目和格狍腔菌 目两个不同的目中, 因此把所具有双囊壁的子囊菌 都归入座囊菌目, 由两个亚目和包括葡萄座腔菌科 在内的 34 科组成, 葡萄座腔菌科内承认 14 个属。之 后的一些学者支持这个观点, 如Sivanesan (1984)把 葡萄座腔菌属和球座菌属都归入座囊菌目下的葡 萄座腔菌科, Hawksworth等(1995)把以葡萄座腔菌 属为代表的 5 个属归入座囊菌目中的葡萄座腔菌 科。这种两个主要分类系统分庭抗礼的局面一直持 续到20世纪90年代末分子系统学的出现(图2)。

\section{3 基于分子系统学的属和种的多样性研究}

DNA序列为探讨真菌界物种、属以至门各级分 类阶元的系统发育关系提供了新的依据。Berbee (1996)利用 18S rRNA的基因序列研究了腔菌纲 (Loculoascomycetes)中各级分类单元之间的系统发 育关系, 然而, 由于所使用的分析方法不同, 葡萄 座腔菌科 2 个属即葡萄座腔菌属和球座菌属的分类 地位变化很大, 在邻接树(neighbour-joining tree)中, 大部分物种与座囊菌目中座囊菌属(Dothidea)的种
类聚在一起, 但在最大似然树(maximum likelihood tree)中, 它们则聚在格孢腔菌目中。在随后的 $18 \mathrm{~S}$ rDNA序列研究中, Silva-Hanlin和Hanlin (1999)无法 确定葡萄座腔菌属、球座菌属等葡萄座腔菌科进化 支应归入座囊菌目还是格狍腔菌目。

Denman等(2000)回顾了葡萄座腔菌属及相关 类群的分类研究历史, 综合形态学和分子系统学的 研究结果, 重新分析了该类真菌的分类依据: 该属 真菌子囊和子囊孢子的特征比较稳定, 但种间子囊 孢子形态相近, 大小重叠, 很难用于划分种, 故主 要依据无性型的形态特征和分子证据来划分种, Kirk等(2001)在Ainsworth \& Bisby's Dictionary of the Fungi (第9版)中记录该科7属69种。鉴于该类真菌的 形态多样化, 许多物种的概念过于宽泛, Crous等 (2006)采用28S rDNA部分序列开展葡萄座腔菌科 的分子系统学研究, 指出广义的葡萄座腔菌属为多 谱系类群, 包括约 20 种, 其无性型对应于当时 18 个 腔孢菌类的属。Kirk等(2008)在Ainsworth \& Bisby's Dictionary of the Fungi (第10版)中记录该科26属 1,517种。

Schoch等(2006)基于座囊菌纲的96个分类单位 的 $18 \mathrm{~S} \mathrm{rRNA} 、 28 \mathrm{~S} \mathrm{rRNA}$ 、翻译延长因子1-alpha (EF1- $\alpha$ )和RNA聚合酶第二大亚基(RPB2)的基因序 列数据构建了多基因系统树。结果表明, 葡萄座腔 菌属和球座菌属的所有成员聚类于一个进化分支, 而与其他目关系疏远。为此建立一个新目, 即葡萄 座腔菌目, 当时仅含葡萄座腔菌科。

近年来, 分子证据大量用于子囊菌的系统分类, 对葡萄座腔菌科及相关类群提出了许多不同的分 类学观点(图2), 承认了葡萄座腔科真菌的10-20个 属不等(Lumbsch \& Huhndorf, 2010; Hyde et al, 2011; Wijayawardene et al, 2012)。根据国际植物分类学会 墨尔本会议修订的《国际藻类真菌及植物命名法 规》和阿姆斯特丹宣言 (Hawksworth et al, 2011; McNeill et al，2012), 采用 “一种真菌，一个名称” 分类命名原则, 无性阶段形态和培养特性纳入真菌 系统分类研究, 葡萄座腔菌科的概念及相关类群归 属不断进行相应的清理与订正 (Kirk et al, 2013)。 依据各属模式标本及相关分子证据特征, Liu等 (2012)列出葡萄座腔科真菌27属, Hyde等(2013, 2014)列出该科28属, 并对该科重要植物病原类群 的系统分类和分子系统学研究进展进行了归纳总 


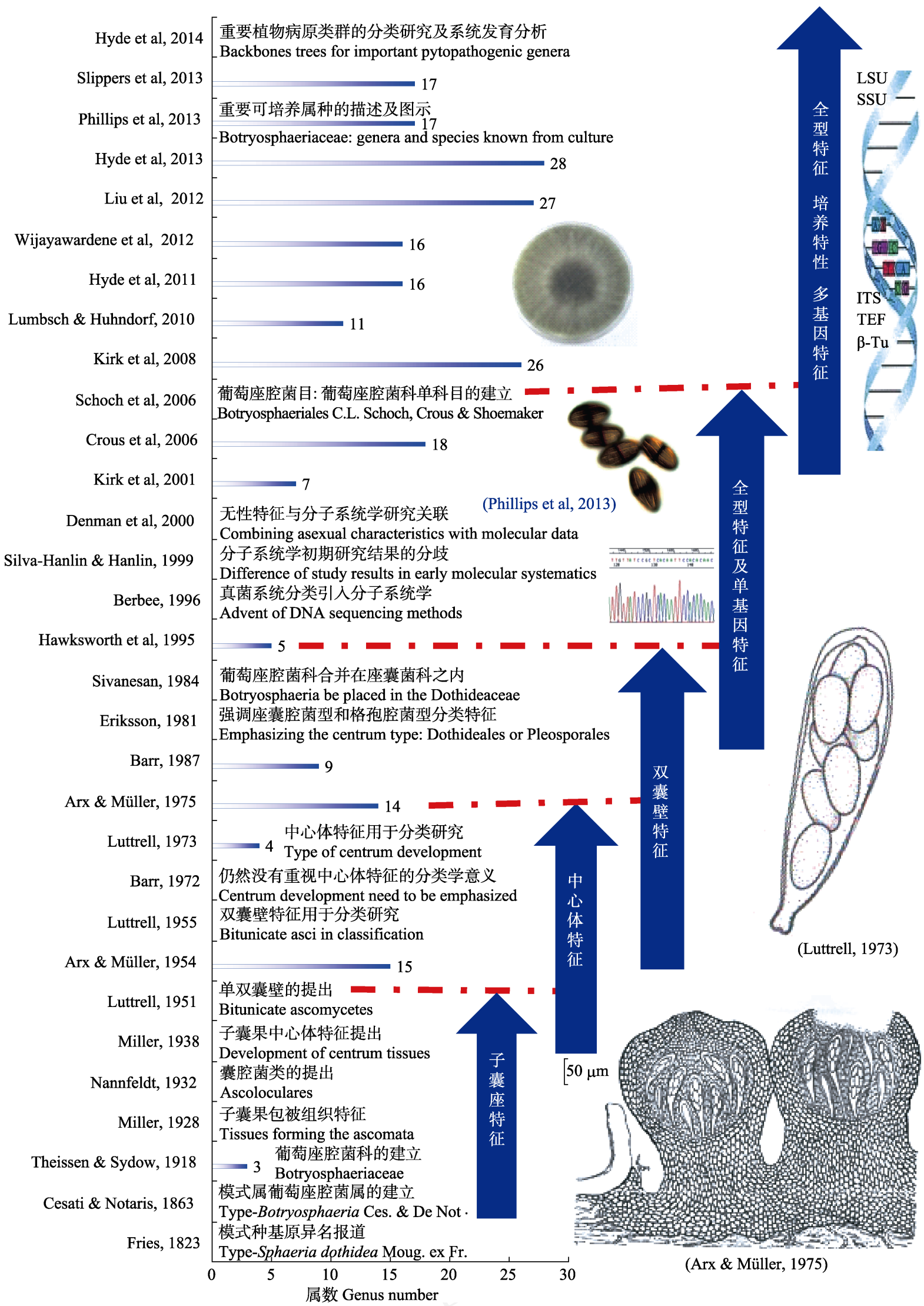

图2葡萄座腔菌科研究进展年代记事表

Fig. 2 The chronicle table on research progresses in Botryosphaeriaceae 
表1 葡萄座腔菌科及其各属研究进展和存在问题

Table 1 Research progresses and comments of genera in Botryosphaeriaceae

\begin{tabular}{lll}
\hline & 存在的问题 Comments & 主要文献 References \\
\hline 葡萄座腔菌科 & 模式科; 属种的分类与名称需要修订 & Cype family, many genera and species need to be cor- \\
Botryosphaeriaceae & rected & 2013; Phillips et al, 2013
\end{tabular}

Barriopsis A. J. L. Phillips et al, 2008 具分子证据, 但标本材料较少

A few molecular data, lack of fresh collections

Botryobambusa R. Phookamsak et al, 2012

\section{葡萄座腔菌属 \\ Botryosphaeria Ces. \& De Not, 1863}

\section{棺狍腔菌属}

Cophinforma Doilom et al, 2012

色二孢属 Diplodia Fr, 1834

小穴壳狍属 Dothiorella Sacc, 1880

Endomelanconiopsis E. I. Rojas \& Samuels, 2008

毛色二狍属

Lasiodiplodia Ellis \& Everh, 1896

亚大茎点菌属

Macrophomina Petr, 1923

Neodeightonia C. Booth, 1970

\section{新壳梭狍属}

Neofusicoccum Crous, 2006

\section{新暗色柱节孢属}

Neoscytalidium Crous \& Slippers, 2006

\section{暗葡腔菌属}

Phaeobotryon Theiss. \& Syd, 1915

\section{拟壳梭孢属}

Pseudofusicoccum Mohali, Slippers \& M. J. Wingf, 2006

斯潘氏菌属

Spencermartinsia A. J. L. Phillips et al, 2008

拟球壳需属

Sphaeropsis Sacc, 1880

Tiarosporella Höhn, 1919
单种属; 竹生, 具分子证据和全型特征

Monotypic genus on bamboo, some molecular data and holomorph characteristics

模式属; 存在复合种, 大量记录名称仍需订正

Type genus, remaining species complex, lots of epithets need to be corrected

具分子证据及全型特征，需更多材料

A few molecular data and holomorph characteristics, lack of more fresh collections

常见属; 大量记录名称需要清理与订正 Common genus, lots of epithets need to be corrected

常见属; 大量记录名称需要订正, 缺乏有性材料

Common genus, lots of epithets need to be corrected, lack of teleomorph collections

具分子证据，缺少有性特征材料

A few molecular data, lack of teleomorph collections

常见属; 具分子证据, 但有性特征材料较少

Common genus, some molecular data, but lack of fresh teleomorph collections

较小属；缺少更多分子证据及有性特征材料

Minor genus, lack of more molecular data and fresh teleomorph collections

新修订属; 具全型特征及分子证据

Revised genus, a few molecular data and holomorph characteristics

常见属; 具分子证据及全型特征

Common genus, a few molecular data and holomorph characteristics

具分子证据, 缺少有性特征材料

A few molecular data, lack of fresh teleomorph collections

新修订属; 具分子证据, 缺少全型特征材料

Revised genus, a few molecular data, lack of fresh holomorph collections

具分子证据，缺少有性特征材料

A few molecular data, lack of fresh teleomorph collections

具较多分子证据，缺乏有型特征材料

Some molecular data, lack of fresh teleomorph collections

常见属; 大量记录名称需要清理与订正

Common genus, lots of epithets need to be corrected

具分子证据, 属内成员需进一步清理与订正

A few molecular data, lots of members need to be corrected
Phillips et al, 2008; Abdollahzadeh et al, 2009; Doilom et al, 2014

Liu et al, 2012; Hyde et al, 2013

Denman et al, 2000; Slippers et al, 2004;

Abdollahzadeh et al, 2013; Phillips et al, 2013

Mehl et al, 2011; Liu et al, 2012; Phillips et al, 2013

Alves et al, 2004, 2014; Phillips et al, 2012, 2013

Crous et al, 2006; Phillips et al, 2008; Phillips et al, 2013; Abdollahzadeh et al, 2014

Rojas et al, 2008; Hyde et al, 2013

Alves et al, 2008; Abdollahzadeh et al, 2010; Phillips et al, 2008, 2013

Crous et al, 2006; Liu et al, 2012; Sarr et al, 2014

Punithalingam, 1969; Phillips et al, 2008, 2013; Liu et al, 2010, 2012; Hyde et al, 2013

Crous et al, 2006; Abdollahzadeh et al, 2013; Phillips et al, 2013; Hyde et al, 2014

Crous et al, 2006; Chuang et al, 2012; Lan et al, 2012; Phillips et al, 2013

Phillips et al, 2008; Abdollahzadeh et al, 2009; Fan et al, 2015

Crous et al, 2006; Pavlic et al, 2008; Mehl et al, 2011

Phillips et al, 2008; Abdollahzadeh et al, 2014

Palmer et al, 1987; Phillips et al, 2008, 2013; Liu et al, 2012; Slippers et al, 2013

Liu et al, 2012; Jami et al, 2012
结。根据真菌全型形态特征、培养特性、分子证据, 结合各类群的分类研究及经济重要性等文献资料, Phillips等(2013)提供了该科可培养17属110种的特 征描述、图示及分种检索表, Slippers等(2013)重新
构建了葡萄座腔菌目系统树, 列出该科28属, 其中 17属提供了分子证据，其余11属还需要对全型特征 和分子证据进行深入研究。Wijayawardene等(2014) 提出座囊菌纲最新分类系统, 其中葡萄座腔菌科为 
葡萄座腔菌目中最大的科, 包括29属, Dissanayake 等(2016)列出该科23属187种的名录并简要讨论, 在 此汇总Phillips等(2013)对具培养特性和分子证据的 17属的分类学处理(表1, 图1,2)。

\section{4 中国葡萄座腔菌类真菌多样性研究}

中国葡萄座腔菌类真菌的研究最早可以追溯 到对贝伦格葡萄座腔菌的报道(Teng, 1934)。早期分 类学研究主要依据有性阶段的形态特征, 20 世纪 50-60年代, 戴芳澜、邓叔群、姜广正、余永年等学 界前辈在全国进行了广泛的真菌资源和植物病害 调查, 收集到许多标本材料, 研究结果收录在真菌 分类研究的文献中。 20 世纪 80 年代后的很长一段时 期, 中国葡萄座腔菌类真菌的分类研究工作一直未 受到重视, 仅有零星报道(黄天章, 1977; 尚衍重, 1987; Sivanesan \& Hsieh, 1989; Hsieh et al, 1994, 1996; Teng, 1996), 缺乏专门研究。《中国的真菌》 中收录葡萄座腔菌类真菌2属3 种(邓叔群, 1963),

《中国真菌总汇》收录2属14种(戴芳澜, 1979), Eriksson和Yue (1988)清理订正并记录2属12种, 《台 湾真菌名录》中收录了分布在台湾省的2属28种(王 也珍等, 1999), Checklist of Hong Kong Fungi中收录 2属9种(Lu et al, 2000)。截至2006年，按照现代分类 学观点, 我国已知葡萄座腔菌类真菌 1 科 5 属 26 种。

近10年来, 笔者与合作者对我国葡萄座腔菌科 开展了系统分类研究, 对保存于中国科学院微生物 研究所真菌标本馆(HMAS)的所有葡萄座腔菌属及 相关类群标本进行了重新鉴定, 按照现代分类学观 点清理和修订了 15 个种, 发表 6 个新种, 2 个中国新 记录种(Li \& Zhuang, 2007)。根据Ainsworth \& Bisby's Dictionary of the Fungi (第10版) (Kirk et al, 2008)的分类观点, 李文英和庄文颖(2013)简述了中 国葡萄座腔菌属的研究进展。结合近年来我国报道 的一些新种和新记录种(李文英, 2008; Zhao, 2010; Chen et al, 2011; Zhou et al, 2016), 对采自全国各地 的 1,000 余份标本材料进行了分类鉴定, 按照现代 分类观点(Liu et al, 2012; Hyde et al, 2013; Phillips et al, 2013; Slippers et al, 2013; Dissanayake et al, 2016), 澄清并确认中国葡萄座腔菌科真菌 12 属 55 种 (表 1 , 图1)。可以预见, 随着研究的深入, 对该类群在我国 分布范围和物种数量将不断刷新。

我国对葡萄座腔菌科真菌的分子生物学研究
起步于对林木溃疡病、果树轮纹病、流胶病等重要 植物病害病原菌的分类地位订正及重要病原菌之 间的系统发育关系等(王金利等, 2007; 赵嘉平等, 2007; 余仲东等, 2010; 李夏等, 2015), 利用我国材 料对葡萄座腔菌科及相关类群的系统学关系进行 了初步探讨, 为建立趋于自然的分类系统提供了有 力佐证(程燕林等, 2011; 王庆灵, 2013 ${ }^{\circledR}$; Fan et al, 2015; Zhou et al, 2016), 如李文英和庄文颖(2009)采 用 $18 \mathrm{~S}$ 和 $28 \mathrm{~S} \mathrm{nrDNA}$ 序列片段对部分葡萄座腔菌科 及相关类群进行了系统发育分析, 提出当时分类地 位不确定的双壁子囊菌大聚颈腔菌属 (Macrovalsaria) 与葡萄座腔菌属的关系比较接近, 应归于葡萄座腔菌科, 这一结果也得到后来学者的 进一步证实和支持(Liu et al, 2012; Hyde et al, 2013; Wijayawardene et al, 2014)。上述研究为进一步探讨 葡萄座腔菌科的属间关系以及深入开展该类真菌 的分子系统学研究提供了科学依据。

\section{5 存在问题及研究展望}

近年来, 真菌分子系统学的研究进展与菌物命 名法规的变革引起真菌分类系统的巨大变化, Wijayawardene等(2014)对葡萄座腔菌类真菌提出新分 类系统, 但学者们普遍认为 Phillips 等 (2013) 和 Dissanayake等(2016)提出的分类学处理得到了分子 证据的支持，更趋于合理。目前该类真菌的研究仍 存在许多悬而未决的问题, 如有些属的分类地位和 命名存在疑问, 有些属中大量名称有待清理与订正, 有些属种缺乏对全型特征的认识, 有些属和种之间 的关系缺乏分子证据等(表1)。因此, 进一步加强资 源挖掘, 深入开展该类真菌的系统分类和物种多 样性研究十分必要。以物种全型的形态特征、培养 特性及多基因序列佐证等作为重要分类依据，是该 类群分类学和系统发育研究的必然趋势。

我国葡萄座腔菌科的分类研究走过了 80 余年 历程, 经过从有性特征分类至全型特征分类、从形 态分类到分子系统研究的发展过程。近年来虽然陆 续报道了一些新种和中国新记录种(表1, 图1), 但已 知种数仅占全球物种数量的 $30 \%$ 左右, 其中许多种 类仍需清理与订正, 物种多样性和地理分布规律、 生态功能和经济重要性等方面尚缺乏全面的研究。

(1)王庆灵 (2013) 葡萄座腔菌科真菌的系统分类研究. 硕士学位论 文, 河北农业大学, 河北保定. 
中国具有多样化的气候与地理类型以及非常 丰富的植被资源, 葡萄座腔菌科真菌丰富的物种多 样性取决于多样化的生境。由于该类群生活史的不 同阶段特征多样, 需要进一步扩大采集范围, 加强 培养特性的研究, 以便获得更多、更全面的信息。 在我国开展葡萄座腔菌科的资源多样性调查、标本 采集、菌株分离培养和系统分类研究, 将进一步加 深我国对该科物种多样性的认识, 为建立趋于自然 的正确属、种概念和明确属间与种间的系统发育关 系及其在具有双囊壁子囊的真菌中的分类地位提 供更加充分的科学依据, 为有益真菌资源的储备及 持续利用奠定基础, 为控制有害真菌的影响提供基 础信息, 将对科学地认识我国生物多样性和生物资 源保护利用起到积极的推动作用。

致谢: 在此文完成过程中, 中国林科院热带林业研 究所梁俊峰研究员、西南大学董国菊博士在文稿修 改中提出许多宝贵意见, 中国科学院微生物研究所 郑焕娣博士、曾昭清博士、陈凯博士在文献提供等 方面给予很大支持和帮助，谨此致谢!

\section{参考文献}

Abdollahzadeh J, Goltapeh EM, Javadi A, Shams-Bakhsh M, Zare R, Phillips AJL (2009) Barriopsis iraniana and Phaeobotryon cupressi: two new species of the Botryosphaeriaceae from trees in Iran. Persoonia, 23, 1-8.

Abdollahzadeh J, Javadi A, Goltapeh EM, Zare R, Phillips AJL (2010) Phylogeny and morphology of four new species of Lasiodiplodia from Iran. Persoonia, 25, 1-10.

Abdollahzadeh J, Javadi A, Zare R, Phillips AJL (2014) A phylogenetic study of Dothiorella and Spencermartinsia species associated with woody plants in Iran, New Zealand, Portugal and Spain. Persoonia, 32, 1-12.

Abdollahzadeh J, Zare R, Phillips AJL (2013) Phylogeny and taxonomy of Botryosphaeria and Neofusicoccum species in Iran, with description of Botryosphaeria scharifii sp. nov. Mycologia, 105, 210-220.

Alves A, Correia A, Luque J, Phillips AJL (2004) Botryosphaeria corticola sp. nov. on Quercus species, with notes and description of Botryosphaeria stevensii and its anamorph, Diplodia mutila. Mycologia, 96, 598-613.

Alves A, Crous PW, Correia A, Phillips AJL (2008) Morphological and molecular data reveal cryptic speciation in Lasiodiplodia theobromae. Fungal Diversity, 28, 1-13.

Alves A, Linaldeddu BT, Deidda A, Scanu B, Phillips AJL (2014) The complex of Diplodia species associated with Fraxinus and some other woody hosts in Italy and Portugal.
Fungal Diversity, 67, 143-156.

Barr ME (1972) Preliminary studies on the Dothideales in temperate North America. Contributions from the University of Michigan Herbarium, 9, 523-638.

Barr ME (1979) A classification of Loculoascomycetes. Mycologia, 71, 935-957.

Barr ME (1983) The ascomycetes connection. Mycologia, 75, $1-13$.

Barr ME (1987) Prodromus to class Loculoascomycetes. Published by the author, Amherst, Massachusetts, USA.

Berbee ML (1996) Loculoascomycete origins and evolution of filamentous Ascomycetes morphology based on 18S rRNA gene sequence data. Molecular Biology and Evolution, 13, 462-470.

Chen SF, Pavlic D, Roux J, Slippers B, Xie YJ, Wingfield MJ, Zhou XD (2011) Characterization of Botryosphaeriaceae from plantation-grown Eucalyptus species in South China. Plant Pathology, 60, 739-751.

Cheng YL, Lv Q, Liang J, Zhang XY (2011) Relationships among fungi of Botryosphaeriaceae causing poplar canker. Forest Pest and Disease, (6), 12-17. (in Chinese with English abstract) [程燕林, 吕全, 梁军, 张星耀 (2011) 杨树 溃疡病病原一葡萄座腔菌科真菌间关系的系统学初探. 中国森林病虫, (6), 12-17.]

Chuang MF, Ni HF, Yang HR, Shu SL, Lai SY, Jiang YL (2012) First report of stem canker disease of pitaya (Hylocereus undatus and $H$. polyrhizus) caused by Neoscytalidium dimidiatum in Taiwan. Plant Disease, 96, 906.

Crous PW, Slippers B, Wingfield MJ, Rheeder J, Marasas WFO, Philips AJ, Alves A, Burgess T, Barber P, Groenewald JZ (2006) Phylogenetic lineages in the Botryosphaeriaceae. Studies in Mycology, 55, 235-253.

Cunnington JH, Priest MJ, Powney RA, Cother NJ (2009) Diversity of Botryosphaeria species on horticultural plants in Victoria and New South Wales. Australasian Plant Pathology, 36, 157-159.

Deng Z, Zhang R, Shi Y, Hu L, Tan H, Cao L (2014) Characterization of Cd-, $\mathrm{Pb}$-, Zn-resistant endophytic Lasiodiplodia sp. MXSF31 from metal accumulating Portulaca oleracea and its potential in promoting the growth of rape in metalcontaminated soils. Environmental Science and Pollution Research, 21, 2346-2357.

Denman S, Crous PW, Taylor JW, Kang JC, Pascoe I, Wingfield MJ (2000) An overview of the taxonomic history of Botryosphaeria, and a re-evluation of its anamorphs based on morphology and ITS rDNA phylogeny. Studies in Mycology, 45, 129-140.

Dissanayake AJ, Phillips AJL, Li XH, Hyde KD (2016) Botryosphaeriaceae: Current status of genera and species. Mycosphere, 7, 1001-1073.

Doilom M, Shuttleworth LA, Roux J, Chukeatirote E, Hyde KD (2014) Barriopsis tectonae sp. nov. a new species of Botryosphaeriaceae from Tectona grandis (teak) in Thailand. 
Phytotaxa, 176, 81-91.

Eriksson OE (1981) The families of bitunicate ascomycetes. Opera Botany, 60, 1-220.

Eriksson OE, Yue JZ (1988) The Pyrenomycetes of China, An Annotated Checklist. Umea, Vasterbotten, Sweden.

Fan XL, Hyde KD, Liu JK, Liang YM, Tian CM (2015) Multigene phylogeny and morphology reveal Phaeobotryon rhois sp. nov. (Botryosphaeriales, Ascomycota). Phytotaxa, 205, 90-98.

Han XM, Liang L, Zhang Z, Li XJ, Yang Y, Meng H, Gao ZH, $\mathrm{Xu} \mathrm{YH}$ (2014) Study of production of sesquiterpenes of Aquilaria senensis stimulated by Lasiodiplodia theobromae. China Journal of Chinese Materia Medica, 39, 192-196. (in Chinese with English abstract) [韩晓敏, 梁良, 张争, 李秀 锦, 杨云, 孟慧, 高志晖, 徐艳红 (2014) 可可毛色二狍 菌对白木香产生倍半萜诱导作用. 中国中药杂志, 39, 192-196.]

Hawksworth DL, Crous PW, Redhead SA, Reynolds DR, Samson RA, Seifert KA, Taylor JW, Wingfield MJ, Abaci O, Aime C, Asan A, Bai FY, de Beer ZW, Begerow D, Berikten D, Boekhout T, Buchanan PK, Burgess T, Buzina W, Cai L, Cannon PF, Crane JL, Damm U, Daniel HM, van Diepeningen AD, Druzhinina I, Dyer PS, Eberhardt U, Fell JW, Frisvad JC, Geiser DM, Geml J, Glienke C, Gräfenhan T, Groenewald JZ, Groenewald M, de Gruyter J, GuéhoKellermann E, Guo LD, Hibbett DS, Hong SB, de Hoog GS, Houbraken J, Huhndorf SM, Hyde KD, Ismail A, Johnston PR, Kadaifciler DG, Kirk PM, Kõljalg U, Kurtzman CP, Lagneau PE, Lévesque CA, Liu X, Lombard L, Meyer W, Miller A, Minter DW, Najafzadeh MJ, Norvell L, Ozerskaya SM, Oziç R, Pennycook SR, Peterson SW, Pettersson OV, Quaedvlieg W, Robert VA, Ruibal C, Schnürer J, Schroers HJ, Shivas R, Slippers B, Spierenburg H, Takashima M, Taşkın E, Thines M, Thrane U, Uztan AH, van Raak M, Varga J, Vasco A, Verkley G, Videira SI, de Vries RP, Weir BS, Yilmaz N, Yurkov A, Zhang N (2011) The Amsterdam declaration on fungal nomenclature. IMA Fungus, 2, 105-112.

Hawksworth DL, Kirk PM, Sutton BC, Pegler DN (1995) Ainsworth \& Bisby's Dictionary of the Fungi, 8th edn. CAB International, Wallingford.

Hsieh WH, Chen CY (1994) Sivanesania, a new botryosphaeriaceous ascomycete genus on Rubus from Taiwan. Mycological Research, 98, 44-46.

Hsieh WH, Chen CY (1996) Validation of Sivanesania. Mycological Research, 100, 1106.

Huang LF, Fang BP, Li KM, Ye SJ, Chen JY, Luo ZX, Wang ZY (2016) First report of Lasiodiplodia theobromae causing a stem canker on sweetpotato in China. Plant Disease, 100, 1948.

Huang TZ (1977) A new species of Botryosphaeria. Acta Microbiologica Sinica, 17, 303-305. (in Chinese with English abstract) [黄天章 (1977) 葡萄座壳属的一个新种. 微生
物学报, 17, 303-305.]

Huang WY, Cai YZ, Hyde KD, Corke H, Sun M (2008) Biodiversity of endophytic fungi associated with 29 traditional Chinese medicinal plants. Fungal Diversity, 33, 61-75.

Hyde KD, Jones EBG, Liu JK, Ariyawansa H, Boehm E, Boonmee S, Braun U, Chomnunti P, Crous PW, Dai DQ, Diederich P, Dissanayake A, Doilom M, Doveri F, Hongsanan S, Jayawardena R, Lawrey JD, Li YM, Liu YX, Lücking R, Monkai J, Muggia L, Nelsen MP, Pang KL, Phookamsak R, Senanayake IC, Shearer CA, Suetrong S, Tanaka K, Thambugala KM, Wijayawardene NN, Wikee S, Wu HX, Zhang Y, Aguirre-Hudson B, Alias SA, Aptroot A, Bahkali A, Bezerra JL, Bhat DJ, Camporesi E, Chukeatirote E, Gueidan C, Hawksworth DL, Hirayama K, Hoog SD, Kang JC, Knudsen K, Li WJ, Li XH, Liu ZY, Mapook A, McKenzie EHC, Miller AN, Mortimer PE, Phillips AJL, Raja HA, Scheuer C, Schumm F, Taylor JE, Tian Q, Tibpromma S, Wanasinghe DN, Wang Y, Xu JC, Yacharoen S, Yan JY, Zhang M (2013) Families of Dothideomycetes. Fungal Diversity, 63, 1-313.

Hyde KD, McKenzie EHC, KoKo TW (2011) Towards incorporating anamorphic fungi in a natural classification - checklist and notes for 2010. Mycosphere, 2, 1-88.

Hyde KD, Nilsson RH, Alias SA, Ariyawansa HA, Blair JE, Cai L, de Cock AWAM, Dissanayake AJ, Glockling SL, Goonasekara ID, Gorczak M, Hahn M, Jayawardena RS, van Kan JAL, Laurence MH, Lévesque CA, Li X, Liu JK, Maharachchikumbura SSN, Manamgoda DS, Martin FN, McKenzie EHC, McTaggart AR, Mortimer PE, Nair PVR, Pawłowska J, Rintoul TL, Shivas RG, Spies CFJ, Summerell BA, Taylor PWJ, Terhem RB, Udayanga D, Vaghefi N, Walther G, Wilk M, Wrzosek M, Xu JC, Yan JY, Zhou N (2014) One stop shop: backbones trees for important pytopathogenic genera: I. Fungal Diversity, 67, 21-125.

Jami F, Slippers B, Wingfield MJ, Gryzenhout M (2012) Five new species of the Botryosphaeriaceae from Acacia karroo in South Africa. Cryptogamie Mycologie, 33, 245-266.

Kirk PM, Cannon PF, David JC, Stalpers JA (2001) Ainsworth $\&$ Bisby's Dictionary of the Fungi, 9th edn. CAB International, Wallingford.

Kirk PM, Cannon PF, Minter DW, Stalpers JA (2008) Ainsworth \& Bisby's Dictionary of the Fungi, 10th edn. CAB International, Wallingford.

Kirk PM, Stalpers JA, Braun U, Crous PW, Hansen K, Hawksworth DL, Hyde KD, Lücking R, Lumbsch TH, Rossman AY, Seifert KA, Stadler M (2013) A without-prejudice list of generic names of fungi for protection under the International Code of Nomenclature for Algae, Fungi and Plants. IMA Fungus, 4, 381-443.

Kumar PS, Singh L (2009) Lasiodiplodia theobromae is a mycoparasite of a powdery mildew pathogen. Mycobiology, 37, 308-309.

Lan GB, He ZF, Xi PG, Jiang ZD (2012) First report of brown 
spot disease caused by Neoscytalidium dimidiatum on $\mathrm{Hy}$ locereus undatus in Guangdong, Chinese Mainland. Plant Disease, 96, 1702.

Li WY (2008) Taxonomy of Dothideales and Botryosphaeriales from China and phylogenic approaches to the related fungi. $\mathrm{PhD}$ dissertation, Institute of Microbiology, Chinese Academy of Sciences, Beijing. (in Chinese with English abstract) [李文英 (2008) 中国座囊菌目和葡萄座腔菌目系统分类 研究. 博士学位论文, 中国科学院微生物研究所, 北京.]

Li WY, Zhuang WY (2007) Re-examinations of Botryosphaeriaceae (Dothideomycetes) from China on deposit in HMAS. Mycotaxon, 100, 209-220.

Li WY, Zhuang WY (2009) Preliminary study on relationships of Dothideales and its allies. Mycosystema, 28, 161-170. (in Chinese with English abstract) [李文英, 庄文颖 (2009) 座 囊菌目及相关类群属间关系的系统学初探. 菌物学报, 28, 161-170.]

Li WY, Zhuang WY (2013) Taxonomy studies on the Botryosphaeria from China. Mycosystema, 32, 108-114. (in Chinese with English abstract) [李文英, 庄文颖 (2013) 中 国葡萄座腔菌属分类研究概况. 菌物学报, 32, 108-114.]

Li X, Li WY, Sun LL, Xu PZ, Lu YS (2015) Analysis of holomorphological characteristics on the pathogen of olecranon peach gummosis in northern Guangdong Province. Chinese Journal of Tropical Crops, 36, 2077-2082. (in Chinese with English abstract) [李夏, 李文英, 孙丽丽, 徐培 智, 卢钰升 (2015) 粤北鹰嘴桃流胶病的病原全型特征 分析. 热带作物学报, 36, 2077-2082.]

Liu JK, Chomnunti P, Cai L, Phookamsak R, Chukeatirote E, Jones EBG, Moslem M, Hyde KD (2010) Phylogeny and morphology of Neodeightonia palmicola sp. nov. from palms. Sydowia, 62, 261-276.

Liu JK, Phookamsak R, Doilom M, Wikee S, Li YM, Ariyawansa H, Boonmee S, Chomnunti P, Dai DQ, Bhat JD, Romero AI, Zhuang WY, Monkai J, Jones EBG, Chukeatirote E, Ko-Ko TW, Zhao YC, Wang Y, Hyde KD (2012) Towards a natural of classification of Botryosphaeriales. Fungal Diversity, 57, 149-210.

Lu BS, Hyde KD, Ho WH, Tsui KM, Taylor JE, Wong KM, Yanna, Zhou DQ (2000) Checklist of Hong Kong Fungi. Fungal Diversity Press, Hong Kong.

Lumbsch HT, Huhndorf SM (2010) Myconet Volume 14. Part One. Outline of Ascomycota-2009. Part Two. Notes on Ascomycete Systematics. Nos. 4751-5113. Fieldiana, Life \& Earth Sciences, 1-64.

Luttrell ES (1951) Taxonomy of the Pyrenomycetes. University of Missouri Studies, Science Series, 24, 1-120.

Luttrell ES (1955) The ascostromatic Ascomycetes. Mycologia, 47, 511-532.

Luttrell ES (1973) Loculoascomycetes. In: The Fungi, Vol. IV A: A Taxonomic Review with Keys (eds Ainsworth GC, Sparrow FK, Sussman AS), pp. 135-219. Academic Press, New York and London.
Machado AR, Pinho DB, Pereira OL (2014) Phylogeny, identification and pathogenicity of the Botryosphaeriaceae associated with collar and root rot of the biofuel plant Jatropha curcas in Brazil, with a description of new species of Lasiodiplodia. Fungal Diversity, 67, 1-17.

McNeill J, Barrie FR, Buck WR, Demoulin V, Greuter W, Hawksworth DL, Herendeen PS, Knapp S, Marhold K, Prado J, Prud'homme Van Reine WF, Smith GF, Wiersema JH, Turland NJ (2012) International Code of Nomenclature for Algae, Fungi, and Plants (Melbourne Code), adopted by the 18th International Botanical Congress Melbourne, Australia, July 2011 electronic. Bratislava: International Association for Plant Taxonomy. [Regnum Vegetabile No. 154.] Königsten: Koeltz Scientific Books.

Mehl JWM, Slippers B, Roux J, Wingfield MJ (2011) Botryosphaeriaceae associated with Pterocarpus angolensis (kiaat) in South Africa. Mycologia, 103, 534-553.

Miller JH (1928) Biologic studies in the Sphaeriales-I. Mycologia, 20, 187-213.

Miller JH (1938) Studies in the development of two Myriangium species and the systematic position of the order Myriangiales. Mycologia, 30, 158-181.

Nannfeldt JA (1932) Studien über die morphologie und systematik der nichtlichenisierten inoperculaten Discomyceten. Nova Acta Regiae Societatis Scientiarum Upsaliensis Series, 4, 8, 1-368.

Palmer MA, Stewart EL, Wingfield MJ (1987) Variation among isolates of Sphaeropsis sapinea in North Central United States. Phytopathology, 77, 944-948.

Parguey-Leduc A (1966) Recherches sur l'ontologénie et l'anátomie comparée des ascocarpes des Pyrénomycètes ascoloculaires. Annales des Sciencies Naturelles. Botanique et Biologie Végétale (París), 7, 505-690.

Pavlic D, Wingfield MJ, Barber P, Slippers B, Hardy GESJ, Burgess TI (2008) Seven new species of the Botryosphaeriaceae from baobab and other native trees in Western Australia. Mycologia, 100, 851-866.

Petrak F (1923) Mycologische Notizen V. No. 200. Über die Pseudosphaeriaceen v.h und ihre Bedeutung für die spezielle Systematik der Pyrenomyzeten. Annales Mycologici, 21, 30-69.

Phillips AJL, Alves A, Abdollahzadeh J, Slippers B, Wingfield MJ, Groenewald JZ, Crous PW (2013) The Botryosphaeriaceae: genera and species known from culture. Studies in Mycology, 76, 51-167.

Phillips AJL, Alves A, Pennycook SR, Johnston PR, Ramaley A, Akulov A, Crous PW (2008) Resolving the phylogenetic and taxonomic status of dark-spored teleomorph genera in the Botryosphaeriaceae. Persoonia, 21, 29-55.

Phillips AJL, Lopes J, Abdollahzadeh J, Bobev S, Alves A (2012) Resolving the Diplodia complex on apple and other Rosaceae hosts. Persoonia, 29, 29-38.

Punithalingam E (1969) Studies on Sphaeropsidales in culture. 
Mycological Papers, 119, 1-24.

Rojas EI, Herre AMLC, Arnold AE, Chaverri P, Samuels GJ (2008) Endomelanconiopsis, a new anamorph genus in the Botryosphaeriaceae. Mycologia, 100, 760-775.

Sarr MP, Ndiaye M, Groenewald JZ, Crous PW (2014) Genetic diversity in Macrophomina phaseolina, the causal agent of charcoal rot. Phytopathologia Mediterranea, 53, 250-268.

Schoch CL, Shoemaker RA, Seifert KA, Hambleton S, Spatafora JW, Crous PW (2006) A multigene phylogeny of the Dothideomycetes using four nuclear loci. Mycologia, 98, 1041-1052.

Shang YZ (1987) Taxonomic study on the pathogen fungus of shoot blight of larch. Acta Mycologica Sinica, 6, 248-249. (in Chinese with English abstract) [尚衍重 (1987) 落叶松 枯梢病菌分类地位的探讨. 真菌学报, 6, 248-249.]

Silva-Hanlin DMW, Hanlin RT (1999) Small subunit ribosomal RNA gene phylogeny of several loculoascomycetes and its taxonomic implications. Mycological Research, 103, 153-160.

Sivanesan A (1984) The Bitunicate Ascomycetes and Their Anamorphs. Cramer, Vaduz, Liechtenstein.

Sivanesan A, Hsieh WH (1989) New species and new records of ascomycetes from Taiwan. Mycological Research, 93, 340-351.

Slippers B, Boissin E, Phillips AJL, Groenewald JZ, Lombard L, Wingfield MJ, Postma A, Burgess T, Crous PW (2013) Phylogenetic lineages in the Botryosphaeriales: a systematic and evolutionary framework. Studies in Mycology, 76, $31-49$.

Slippers B, Crous PW, Denman S, Coutinho TA, Wingfield BD, Wingfield MJ (2004) Combined multiple gene genealogies and phenotypic characters differentiate several species previously identified as Botryosphaeria dothidea. Mycologia, 96, 83-101.

Slippers B, Smit WA, Crous PW, Coutinho TA, Wingfield BD, Wingfield MJ (2007) Taxonomy, phylogeny and identification of Botryosphaeriaceae associated with pome and stone fruit trees in South Africa and other regions of the world. Plant Pathology, 56, 128-139.

Slippers B, Wingfield MJ (2007) Botryosphaeriaceae as endophytes and latent pathogens of woody plants: diversity, ecology and impact. Fungal Biology Reviews, 21, 90-106.

Tai FL (1979) Sylloge Fungorum Sinicorum. Science Press, Beijing. (in Chinese) [戴芳澜 (1979) 中国真菌总汇. 科学 出版社, 北京.]

Teng SC (1934) Notes on Sphareiales from China. Sinensia, 4, 389-390.

Teng SC (1963) Fungi of China. Science Press, Beijing. (in Chinese) [邓叔群 (1963) 中国的真菌. 科学出版社, 北京.]

Teng SC (Korf RP ed.) (1996) Fungi of China. Mycotaxon Ltd., Ithaca.

Theissen F, Sydow H (1918) Vorentwürfe zu den Pseudosphaeriales. Annales Mycologici, 16, 1-34. von Arx JA, Müller E (1954) Die Gattungen der amerosporen Pyrenomyceten. Beiträge zur Kryptogamenflora der Schweiz, 11, 1-434.

von Arx JA, Müller E (1975) A re-evaluation of the bitunicate ascomycetes with keys to families and genera. Studies in Mycology, 9, 1-159.

Wang JL, He W, Qin GF, Tao WQ, Zhao J, Tian SM (2007) Study on the anamorph and species of Botryosphaeria, the important pathogen of tree's canker. Forest Research, 20(1), 21-28. (in Chinese with English abstract) [王金利, 贺伟, 秦国夫, 陶万强, 赵俊, 田淑敏 (2007) 树木溃疡病重要 病原葡萄座腔菌属、种及其无性型研究. 林业科学研究, 20(1), 21-28.]

Wang YZ, Wu SH, Zhou WN, Chang DZ, Chen KY, Chen SF, Chen CL, Tzeng SS, Liou JH, Hsieh WH, Hsieh HR, Chung $\mathrm{CH}$, Chien CY (eds) (1999) List of the Fungi in Taiwan. Committee for Agriculture, Taipei. (in Chinese) [王也珍, 吴声华, 周文能, 张东柱, 陈桂玉, 陈淑芬, 陈城箖, 曾 须雄，刘锦惠，谢文瑞，谢焕儒，钟兆玄，简秋源 (1999) 台湾真菌名录. “行政院”农业委员会, 台北.]

Wei W, Jiang N, Mei YN, Chu YL, Ge HM, Song YC, Ng SW, Tan RX (2014) An antibacterial metabolite from Lasiodiplodia pseudotheobromae F2. Phytochemistry, 100, 103109.

Wijayawardene NN, Crous PW, Kirk PM, Hawksworth DL, Boonmee S, Braun U, Dai DQ, D'souza MJ, Diederich P, Dissanayake A, Doilom M, Hongsanan S, Gareth Jones EB, Groenewald JZ, Jayawardena R, Lawrey JD, Liu JK, Lücking R, Madrid H, Manamgoda DS, Muggia L, Nelsen MP, Phookamsak R, Suetrong S, Tanaka K, Thambugala KM, Wanasinghe DN, Wikee S, Zhang Y, Aptroot A, Ariyawansa HA, Bahkali AH, Bhat DJ, Gueidan C, Chomnunti P, De Hoog GS, Knudsen K, Li WJ, McKenzie EHC, Miller AN, Phillips AJL, Piątek M, Raja HA, Shivas RS, Slippers B, Taylor JE, Tian Q, Wang Y, Woudenberg JHC, Cai L, Jaklitsch WM, Hyde KD (2014) Naming and outline of Dothideomycetes-2014 including proposals for the protection or suppression of generic names. Fungal Diversity, 69, 1-55.

Wijayawardene NN, McKenzie EHC, Hyde KD (2012) Towards incorporating anamorphic fungi in a natural classification-checklist and notes for 2011. Mycosphere, 3, 157-228.

Wu XQ, He YQ, Liu ZH (2001) Occurrence and progress on tree cankers caused by Botryosphaeria spp. Journal of Nanjing Forestry University, 25, 61-66. (in Chinese with English abstract) [吴小芹, 何月秋, 刘忠华 (2001) 葡萄座腔 菌属所致树木溃疡病发生与研究进展. 南京林业大学学 报, 25(1), 61-66.]

Xu C, Wang C, Ju L, Zhang R, Biggs AR, Tanaka E, Li B, Sun G (2015a) Multiple locus genealogies and phenotypic characters reappraise the causal agents of apple ring rot in China. Fungal Diversity, 71, 215-231.

Xu C, Zhang H, Zhou Z, Hu T, Wang S, Wang Y, Cao K (2015b) Identification and distribution of Botryosphaeriaceae 
species associated with blueberry stem blight in China. European Journal of Plant Pathology, 143, 1-16.

Yu ZD, Zhao GC, Dan JY, Ren ZZ (2010) Phylogeny of Botryosphaeria species based on ITS-nrDNA sequences. Mycosystema, 29, 285-293. (in Chinese with English abstract) [余仲东, 赵官成, 淡静雅, 任争争 (2010) 葡萄座腔菌属 ITS-nrDNA的分子系统学分析. 菌物学报, 29, 285-293.]

Zhang ZY, Wang CP, Liu HB, Sun HW (2012) Bioremediation of PAHs contaminated coil from Beijing coking plant by Lasiodiplodia theobromae. Environmental Science, 33, 2832-2839. (in Chinese with English abstract) [张志远, 王 翠苹, 刘海滨, 孙红文 (2012) 可可毛色二狍菌对焦化厂 土壤多环芳烃污染修复. 环境科学, 33, 2832-2839.]

Zhao JP, Liang J, Lv Q, Zhang XY (2007) Review on the systemic taxonomy of genus Botryosphaeria. Forest Research, 20, 415-422. (in Chinese with English abstract) [赵嘉平,
梁军, 吕全, 张星耀 (2007) 葡萄座腔菌属系统分类评 述. 林业科学研究, 20, 415-422.]

Zhao JP, Lü Q, Liang J, Decock C, Zhang XY (2010) Lasiodiplodia pseudotheobromae, a new record of pathogenic fungi from some subtropical and tropical trees in southern China. Cryptogamie Mycologie, 31, 431-439.

Zhou Y, Dou Z, He W, Zhang X, Zhang Y (2016) Botryosphaeria sinensia sp nov., a new species from China. Phytotaxa, 245, 43-50.

Zhou Y, Gong G, Cui Y, Zhang D, Chang X, Hu R, Liu N, Sun X (2015) Identification of Botryosphaeriaceae causing kiwifruit rot in Sichuan Province, China. Plant Disease, 99, 699-708. 